\title{
Dropped Head Syndrom als Folge einer behandelbaren metabolischen Myopathie
}

\section{Dropped Head Syndrome Caused by a Treatable Metabolic Myopathy}

\begin{abstract}
WARUM DIESER FALL?
Wir berichten über den ungewöhnlichen Fall einer metabolischen Myopathie, die sich mit einem Dropped Head Syndrom (DHS) mit myographischen und bildgebenden Veränderungen vereinbar mit einer fokalen inflammatorischen Myopathie erstmanifestierte. Der Fall zeigt, dass die gezielte Biopsie auch schwerer zugänglicher Muskeln entscheidend sein kann zur korrekten Diagnose.
\end{abstract}

Schwäche der Kopfhaltemuskulatur ist die Folge einer Schädigung der zervikothorakalen Paravertebralmuskulatur oder der sie versorgenden Motoneurone. Die häufigsten neuromuskulären Erkrankungen, die ein DHS verursachen, sind die amyotrophe Lateralsklerose, entzündliche Myopathien und die Myasthenie [1]. Zusätzlich gibt es jedoch eine ganze Reihe weiterer neuromuskulärer Erkrankungen, die zu eine isolierten oder schwerpunktmäßig die Kopfhaltemuskulatur betreffenden Schwäche führen können ( $\triangleright$ Tab. 1). In einigen Fällen - v. a. bei älteren Patienten - bleibt die Ursache des DHS ungeklärt und wird auch als isolierte Nackenstreckermyopathie beschrieben [2]. Auch neurodegenerative Erkrankungen wie das idiopathische Parkinson-Syndrom oder die Multisystematrophie können zu einem DHS führen [3, 4]. Im hier beschriebenen Fall ist die Ursache eine Fettsäureoxidationsstörung mit Symptombeginn im Erwachsenenalter, dem multiplen Acyl-CoA-Dehydrogenase-Mangel (MADD). Die Diagnosestellung ist v. a. deshalb relevant, weil es sich um eine behandelbare Stoffwechselstörung handelt, die unbehandelt zu schweren Komplikationen wie einer Rhabdomyolyse führen kann.

\section{Anamnese}

Die 63-jährige Büroangestellte stellt sich vor wegen einer seit 6 Wochen bestehen- den Kopfhalteschwäche. Sie kann sich nur noch mit Abstützen der Arme aufrecht halten. Begleitende Muskelschmerzen oder Schwäche der Extremitäten werden verneint, die Schwäche führt aber zu einer erheblichen Beeinträchtigung der Alltagsaktivitäten. Die Patientin hat einen muskelgesunden 31-jährigen Sohn, die Eltern sind muskelgesund und nicht verwandt. Eine vorausgehende Episode erhöhter CK und unklarer Muskelschwäche remittierte unter einer passageren oralen Steroidtherapie.

\section{Untersuchungsbefund}

Die Sprache ist klar artikuliert, Okulomotorik und mimische Muskulatur sind unauffällig. Der Kopf fällt beim Aufstehen nach vorne mit ausgeprägter Kyphosierung der oberen Brustwirbelsäule ( $\mathbf{A b b}$. 1). Beim Gehen muss sich die Patienten mit den Händen an den Oberschenkeln oder am Rollator abstützen. Die Kraftprüfung ergibt für die Kopfstrecker MRC-Grad 2, für die Beuger und die proximalen Extremitätenmuskeln Grad 4 + . Das Atemmuster ist im Liegen normal mit kräftigem Hustenstoß und einer Atemfrequenz von 16/min. Die internistische Untersuchung ergibt keinen pathologischen Befund.

\section{Zusatzuntersuchungen}

Die CK ist mit 2480 U/l auf das 15-fache des oberen Normwertes erhöht, die LDH geringer mit 1037 U/I (obere Normgrenze 214). Die GGT ist ebenfalls deutlich induziert mit 180 U/I (Normgrenze 60), ebenso die Transaminasen mit einer GPT von $110 \mathrm{U} / \mathrm{l}$ und einer GOT von 125 U/I (Normgrenzen 50). Die Lipase ist grenzwertig mit 88U/I (Normgrenze 80). Normwertig sind Bilirubin, BSG, CRP, Blutbild. HMG-CoA-Reduktase-Antikörper sind nicht nachweisbar.

Das EMG der zervikalen Paravertebralmuskulatur zeigt eine pathologisch verlängerte Insertionsaktivität, Fibrillationen und positive scharfe Wellen an mehreren Insertionsstellen. Motorische Einheiten sind ver- mehrt niedrigamplitudig und polyphasisch ( $\triangleright$ Abb. 1b). Im Gluteus medius sind myogene Veränderungen geringer ausgeprägt.

Das Magnetresonanztomogramm (MRT) der Nackenregion zeigt etwas schmächtige zervikothorakale Paravertebralmuskeln mit prominenten Bindegewebssepten. In den fettsupprimierten STIR-Sequenzen fallen strähnige Flüssigkeitseinlagerungen in der Paravertebralmuskulatur auf, die Schultermuskulatur stellt sich signalunauffällig dar ( $\triangleright$ Abb. 2a, b). Das MRT der Oberschenkel ergibt ebenfalls einen Normalbefund.

Da der klinische Schwerpunkt der Paresen und die bildgebenden Auffälligkeiten auf die Paravertebralmuskulatur beschränkt sind, wird eine Biopsie des Erector spinae im oberen Thorakalbereich im Rahmen einer Propofol-Kurznarkose durchgeführt.

\section{Verlauf}

Bei der Verdachtsdiagnose einer autoimmunvermittelten paravertebralen Myositis wird unmittelbar im Anschluss an die Biopsie in Kurznarkose eine 5-tägige Steroidpulstherapie mit $750 \mathrm{mg}$ alpha-MethylprednisoIon Tagesdosis durchgeführt. Es kommt darunter zunächst zu einer erheblichen Zunahme der Muskelschwäche jetzt auch der proximalen Extremitäten, sodass die Patientin nicht mehr ohne Hilfe das Bett verlassen kann und beim begleiteten Gehen mit Rollator ein ausgeprägtes TrendelenburgZeichen zeigt. Die Kopfhalteschwäche persistiert. Die CK steigt an auf 11700 U/I (70 x obere Normgrenze, Norm <167) an, die LDH auf $3571 \mathrm{U} / \mathrm{l}$ (Norm <214). Es kommt auch zu einer gering erhöhten Myoglobinausscheidung im Urin $(38 \mathrm{ng} / \mathrm{ml}$, Norm <20) im Rahmen der Rhabdomyolyse. Nach 3 Wochen ist die CK nur noch gering erhöht mit $345 \mathrm{U} / \mathrm{l}$, die LDH noch deutlicher mit $731 \mathrm{U} / \mathrm{l}$. Der weitere klinische Verlauf ist dann gekennzeichnet durch eine allmähliche Besserung der Kraft. Während einer anschließenden stationären Rehabilitationsbehandlung kompliziert eine Pankreatitis die Rekonvaleszenz. Unter Supplementation mit Riboflavin 
- Tab. 1 Differenzialdiagnose des Dropped Head Syndroms.

\begin{tabular}{|c|c|}
\hline Erkrankung & Diagnose \\
\hline \multirow[t]{3}{*}{ Systemerkrankungen } & Idiopathisches Parkinson-Syndrom \\
\hline & Multisystematrophie \\
\hline & Amyotrophe Lateralsklerose \\
\hline Neuropathien & CIDP \\
\hline \multirow[t]{3}{*}{ Endplattenerkrankungen } & Myasthenia gravis \\
\hline & Kongenitale Myasthenien \\
\hline & Myasthenes Syndrom (LEMS) \\
\hline \multicolumn{2}{|l|}{ Myopathien } \\
\hline \multirow[t]{6}{*}{ a) entzündlich } & Steroidresponsive fokale Myositis \\
\hline & Myositis-Scleroderma-Overlap \\
\hline & Brachiozervikale inflamm.Myop. \\
\hline & Dermatomyositis \\
\hline & Vaskulitis (nichtsystemisch) \\
\hline & Einschlusskörpermyositis \\
\hline \multirow[t]{9}{*}{ b) nicht entzündlich } & Idiopathisch \\
\hline & FSHD \\
\hline & Myotone Dystrophie \\
\hline & Proximale myotone Myopathie \\
\hline & Okulopharyngeale Muskeldystrophie \\
\hline & Adulte Nemalin-Myopathie \\
\hline & $1^{\circ}$ Amyloidose (MGUS-assoziiert) \\
\hline & Kongenitale Myopathien (LMNA, SEPN1, MYH7) \\
\hline & VCP-Mutation \\
\hline \multirow[t]{6}{*}{ c) Metabolisch } & Mitochondriale Myopathie \\
\hline & MADD \\
\hline & Carnitin-Mangel \\
\hline & Hypothyreote Myopathie \\
\hline & Hypokaliämische Myopathie \\
\hline & Hyperparathyreoidismus \\
\hline \multirow[t]{3}{*}{ d) Toxisch/iatrogen } & Radiatio des oberen Mantelfeldes \\
\hline & MEK-Inhibitor (Selumetinib) \\
\hline & Dorsale Laminektomie der HWS \\
\hline Fehlbildung & Syringomyelie \\
\hline Mechanisch & Hyperflexions-Trauma \\
\hline
\end{tabular}

250 mg, Coenzym Q10 120 mg und L-Carnitin $3 \times 1 \mathrm{~g}$ tgl. kommt es allmählich zu einer kompletten Remission der schweren Paresen innerhalb von 3 Monaten.

\section{Weiterführende Untersuchungen}

Die Muskelbiopsie der Paravertebralmuskulatur ( $\triangleright$ Abb. 3 ) zeigt vereinzelte Einzelfasernekrosen, vereinbar mit einer nekroti-
Die Untersuchung der Plasma-Acylcarnitine zeigt deutlich erhöhte Konzentrationen fast aller kurz-, mittel- und langkettigen Acylcarnitine, die Analyse des Urins auf organische Säuren ergibt mäßig erhöhte, in dieser Konstellation aber eindeutig pathologische Ausscheidungen von Ethylmalonsäure, Glutarsäure, 2-OH-Glutarsäure, sowie der Dicarbonsäuren Adipin-, Suberinund Sebacinsäure und der Acylglycine Hexanoyl- und Suberylglycin. Gesamt- und freies Carnitin im Serum sind unfauffällig. Die Konstellation ist dringend verdächtig auf einen multiplen Acyl-CoA-Dehydrogenase-Mangel,, der verursacht werden kann durch Mutationen im ETFDH, ETFA- oder ET$F B$-Gen. Die molekulargenetische Paneldiagnostik ergibt 2 pathogene Varianten im ETFDH-Gen: c.241C>T; p.Leu81Phe und c.1571C > T; p.Leu524Pro, die die biochemische Verdachtsdiagnose unterstützen, compound Heterozygotie vorausgesetzt.

\section{Diskussion}

Das subakute Auftreten einer ausgeprägten Kopfhalteschwäche und die Anamnese einer steroidresponsiven Schwächeepisode legten differenzialdiagnostisch eine entzündliche Myopathie nahe, wie sie z. B. im Rahmen von Overlap-Syndromen nahezu isoliert mit einer Schwäche der Paravertebralmuskulatur auftreten kann $[5,6]$ oder auch ohne Begleiterkrankung als fokale steroidresponsive Myositis [7]. EMG und Muskel-MRT wurden zunächst als vereinbar mit dieser Diagnose gesehen. Erst die Muskelbiopsie der betroffenen Paravertebralmuskulatur ergab mit dem Nachweis von Lipidvakuolen den Hinweis auf eine mögliche metabolische Myopathie.

Der Fall illustriert, dass die muskelbioptische Diagnostik beim Verdacht auf entzündliche Myopathien weiterhin indiziert bleibt, da in diesem Fall die erst in der Biopsie nachweisbare abnorme Lipidspeicherung den ersten Hinweis auf eine mögliche metabolische Muskelerkrankung ergab. Zwar erfordert die Biopsie der Paravertebralmuskulatur eine Kurznarkose wegen der notwendigen tiefen Präparation, eine Biopsie eines klinisch nicht betroffenen Muskels wäre aber bei der Verdachtsdiagnose einer fokalen entzündliche Myopathie mit einem hohen Risiko eines falsch negativen Befundes verbunden und es bleibt auch fraglich, ob die pathologische Lipidspeicherung in 

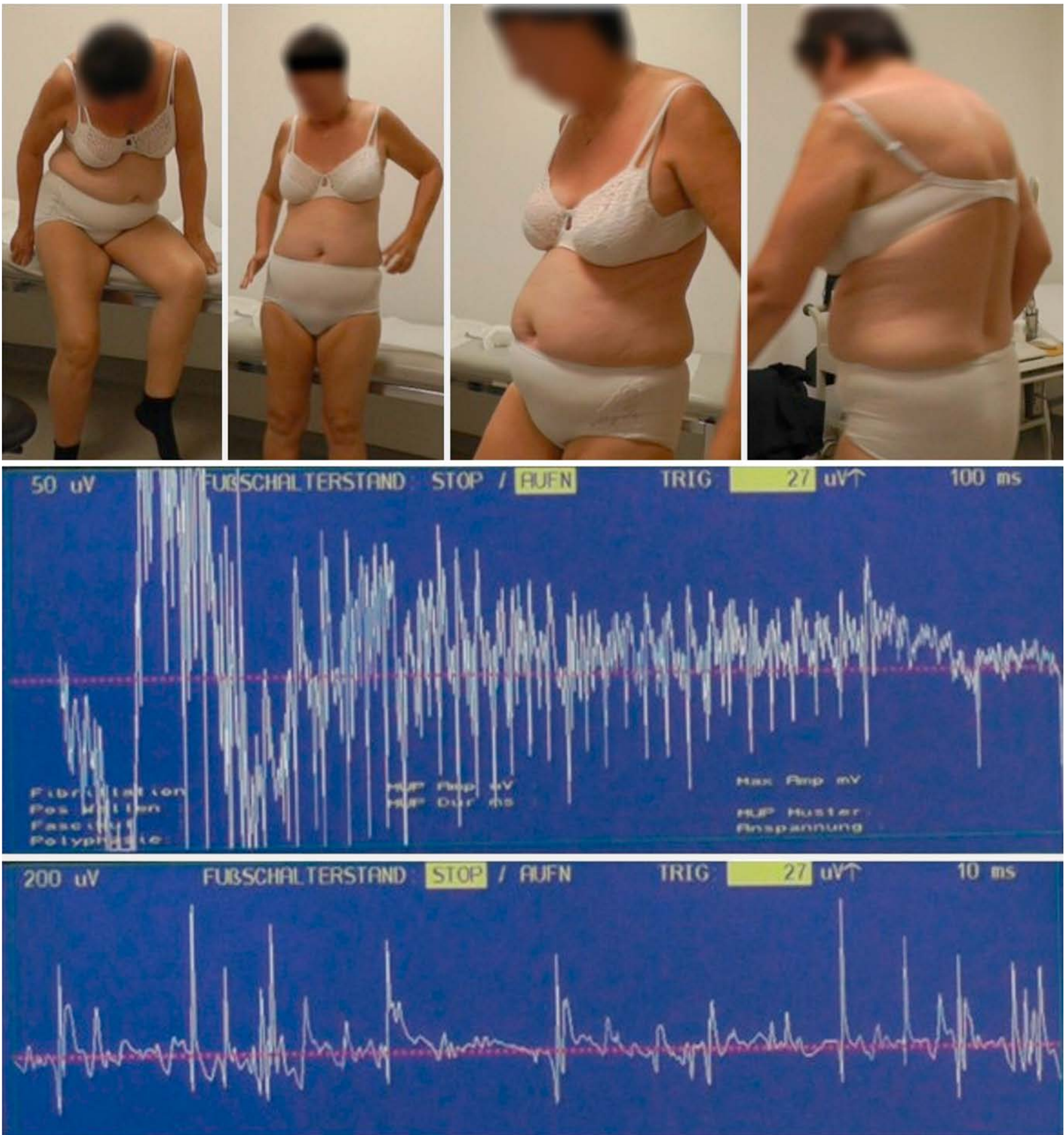

- Abb. 1 Beim Aufstehen und Gehen bleibt der Kopf gebeugt, der Rumpf leicht rekliniert mit Verstärkung der thorakalen Kyphose. Die Kopfaufrichtung gelingt nur kurzzeitig und ist nicht vollständig (oberer Abbildungsteil; 2. Bild bon links). Das EMG (unterer Abbildungsteil) der zervikalen Paravertebralmuskulatur zeigt eine verlängerte Insertionsaktivität (oben) und kurze niedrigamplitudige motorische Einheiten bei Willkürinnervation (unten).

einem klinisch nicht betroffenen Muskel ebenfalls hätte identifiziert werden können. Bei einer anderen metabolischen Myopathie, dem sauren alpha-Glucosidase-Mangel (M.Pompe) kann die Biopsie nicht betroffener Muskeln Normalbefunde ergeben, obwohl es sich dabei ebenfalls um einen in allen Muskelzellen vorhandenen Defekt handelt [8].
Der weitere klinische Verlauf, insbesondere die Rhabdomyolyse nach der Kurznarkose und die Entwicklung einer generalisierten schweren proximalen Muskelschwäche ist dagegen eher mit einer metabolischen Störung v. a. vom Typ der Fettsäureoxidationsstörungen vereinbar als mit einer entzündlichen Myopathie. Die häufigste Lipidstoffwechselstörung mit myopathischem Muster ist 

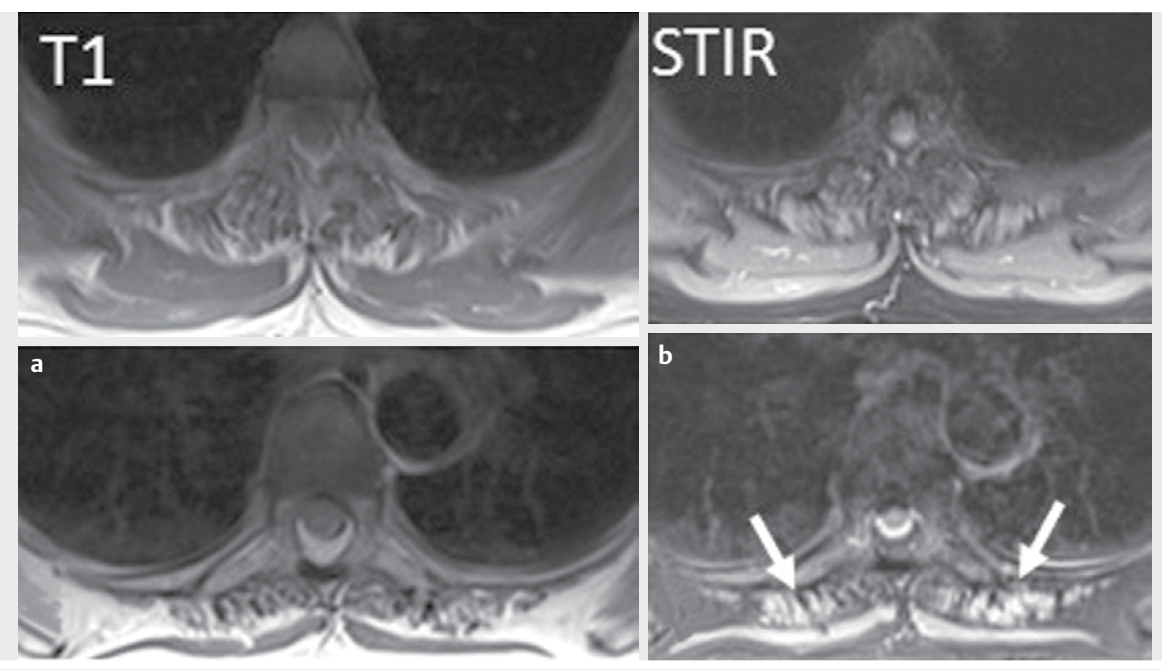

- Abb. 2 Kernspintomogramm des zervikothorakalen Übergangs: Linke Spalte oben und unten a axial T1. STIR fettsupprimiert. Die STIR-Sequenzen zeigen eine strähnige Signalanhebung in der Paravertebralmuskulatur (Pfeile) vereinbar mit vermehrter Flüssigkeitseinlagerung. Auch die axial mit dargestellten Trapeziusanteile sind signalangehoben.
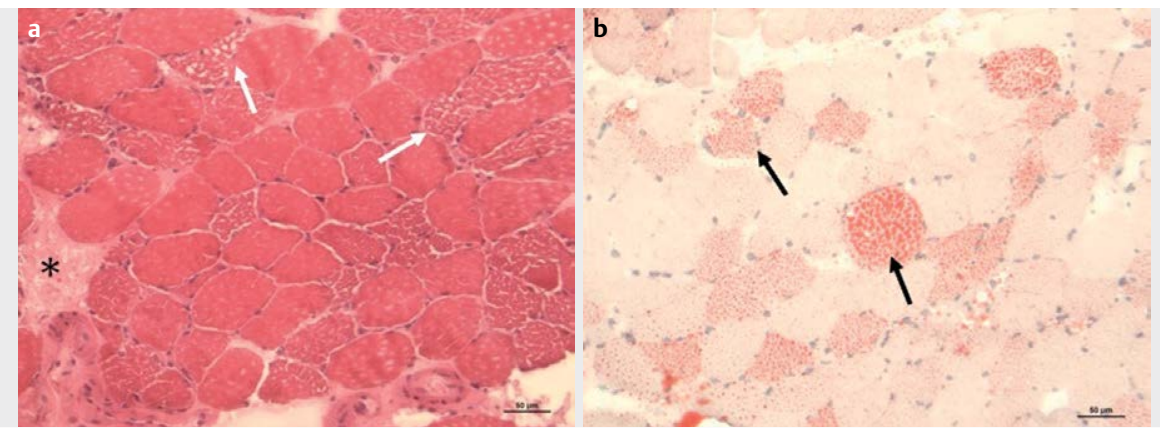

- Abb. 3 Muskelbiopsie der thorakalen Paravertebralmuskulatur. a HE-Färbung: Mehrere Fasern mit klein- bis mittelgroßen vakuolären Einschlüssen (Pfeil) und einzelne nekrotische Fasern. b Ölrot-O-Färbung Fasern mit unterschiedlich großen Lipidtropfen.

dabei der Carnitin-Palmityl-Transferase-II-Mangel, der zu Rhabdomyolysen typischerweise nach Ausdauerbelastungen, aber auch bei prolongiertem Fasten und nach Infekten führen kann [9]. Der bei dieser Patientin diagnostizierte multiple Acyl-CoA-Dehydrogenase-Mangel (MADD, syn. Glutarazidurie Typ IIC) wird verursacht durch compound heterozygote oder homozygote Mutationen der mitochondrialen Elektronentransfer-Flavoprotein Dehydrogenase ETFDH, wobei das ETFDH-Gen nukleär lokalisiert ist auf Chromosom 4q32 (OMIM * 231675). Vergleichbare klinische Phänotypen können auch bei Mutationen der beiden anderen beteiligten Komponenten des Flavoprotein-Elektronentransports auftreten (ETFA und ETFB). Mutationen in diesen Genen können zu schweren hepatischen Enzephalopathien im Kleinkindesalter führen mit fazialer Dysmorphie, fataler neonataler Azidose und Hypoglykämien, die milderen kindlichen Verläufe führen zu episodischem Erbrechen mit Hypoglykämien und Rhabdomyolysen. Die spät beginnende myopathische Form des MADD führt dagegen zu Belastungsintoleranz und fluktuierenden Schwächeepisoden mit erhöhter CK und auch zu Rhabdomyolysen, z. T. getriggert durch Infekte, Fasten, operative Eingriffe, wie z. B. die Muskelbiopsie mit Propofol-Kurz- narkose in diesem Fall. Dabei stehen proximale und axiale Paresen im Vordergrund, insbesondere auch eine Kopfhalteschwäche bzw. ein DHS [10-12].

\section{Zusammenfassung}

Die Diagnosestellung der multiplen Acyl-CoA Dehydrogenase-Mangels (MADD, syn. Glutarazidurie Typ IIc) ist relevant wegen der therapeutischen Konsequenzen: Die Erkrankung bzw. die Muskelschwäche lässt sich bessern durch eine Riboflavin- bzw. Coenzym Q10-Behandlung. Sie ist somit eine der wenigen metabolischen Myopathien für die eine Supplementationstherapie indiziert ist und die zu einer Wiederherstellung der Muskelkraft führen kann [13]. Alternativ wird auch der Einsatz von Riboflavin und L-Carnitin empfohlen[10]. Unter einer Behandlung mit $250 \mathrm{mg}$ Riboflavin, $120 \mathrm{mg}$ Coenzym $\mathrm{Q}$ und L-Carnitin $3 \times 1 \mathrm{~g}$ Tagesdosis bildete sich die hochgradige Tetraparese im vorliegenden Fall innerhalb von 3 Monaten komplett zurück. Die Diagnose kann über eine Bestimmung der Acylcarnitine im Trockenblut oder im Plasma und die Bestimmung der organischen Säuren im Urin oder über eine Muskelbiopsie aufgrund der Lipidspeicherung im Muskel vermutet werden, die Dia- 
gnosebestätigung erfolgt molekulargenetisch. Bei Fällen unklarer Rhabdomyolysen sollten daher das Acylcarnitinprofil im Trockenblut und Plasma und organische Säuren im Urin bestimmt werden, ebenso bei einem Dropped Head Syndrom, wenn dessen Ursache nicht von vorneherein einer anderen Erkrankung zugeordnet werden muss.

\section{WAS LERNT MAN DARAUS?}

Der multiple Acyl-CoA-Dehdrogenase-Mangel (MADD, Glutarazidurie IIc) kann sich im Erwachsenenalter erstmanifestieren als ein subakutes Dropped Head Syndrom oder auch mit proximaler Muskelschwäche, erhöhter CK bis hin zur Rhabdomyolyse bei zuvor asymptomatischen Patienten. Exazerbationen werden getriggert durch Fasten, Infekte, operative Eingriffe und außergewöhnliche körperliche Belastungen. MADD kann - wie auch andere mit Rhabdomyolysen assoziierte Fettsäureoxidationsstörungen - durch biochemische Untersuchungen (Acylcarnitinprofil und Bestimmung organischer Säuren im Urin) diagnositziert und bei pathologischem Befund auch molekulargenetisch gesichert werden. Die Diagnose ist relevant, da eine Therapie mit Riboflavin und Coenzym Q10 oder L-Carnitin zu einer kompletten Remission führen kann.

Interessenkonflikt

Die Autoren geben an, dass kein Interessenkonflikt besteht.
Literatur

[1] Lange DJ, FM Lovelace RE et al. The floppy head syndrome [Abstract]. Ann Neurol 1986; 20: 133

[2] Katz JS, Wolfe GI, Burns DK et al. Isolated neck extensor myopathy: a common cause of dropped head syndrome. Neurology 1996; 46: 917-921

[3] Askmark H, Eeg-Olofsson K, Johansson A et al. Parkinsonism and neck extensor myopathy: a new syndrome or coincidental findings? Archives of neurology 2001; 58: 232-237

[4] Savica R, Kumar N, Ahlskog JE et al. Parkinsonism and dropped head: dystonia, myopathy or both? Parkinsonism \& related disorders 2012; 18: 30-34

[5] Garcin B, Lenglet T, Dubourg O et al. Dropped head syndrome as a presenting sign of scleromyositis. Journal of the neurological sciences 2010; 292: 101-103

[6] Rosato E, Rossi C, Salsano F. Dropped head syndrome and Systemic sclerosis. Joint Bone Spine 2009; 76: 301-303

[7] Kastrup A, Gdynia HJ, Nagele T et al. Dropped-head syndrome due to steroid responsive focal myositis: a case report and review of the literature. Journal of the neurological sciences 2008; 267: 162-165

[8] Muller-Felber W, Horvath R, Gempel K et al. Late onset Pompe disease: clinical and neurophysiological spectrum of 38 patients including long-term follow-up in 18 patients. Neuromuscular disorders: NMD 2007; 17: 698-706

[9] Deschauer M, Wieser T, Zierz S. Muscle carnitine palmitoyltransferase II deficiency: clinical and molecular genetic features and diagnostic aspects. Archives of neurology 2005; 62: 37-41

[10] Behin A, Acquaviva-Bourdain C, Souvannanorath $S$ et al. Multiple acyl-CoA dehydrogenase deficiency (MADD) as a cause of late-onset treatable metabolic disease. Revue neurologique 2016; 172: 231-241

[11] Wen B, Dai T, Li W et al. Riboflavin-responsive lipid-storage myopathy caused by ETFDH gene mutations. Journal of neurology, neurosurgery, and psychiatry 2010; 81 : 231-236
[12] Liang WC, Ohkuma A, Hayashi YK et al. ETFDH mutations, CoQ10 levels, and respiratory chain activities in patients with riboflavin-responsive multiple acyl-CoA dehydrogenase deficiency. Neuromuscular disorders: NMD 2009; 19: 212-216

[13] Gempel K, Topaloglu H, Talim B et al. The myopathic form of coenzyme Q10 deficiency is caused by mutations in the electron-transferring-flavoprotein dehydrogenase (ETFDH) gene. Brain: a journal of neurology 2007; 130: 2037-2044

\section{Autoren}

Bertold Schrank' ${ }^{1}$ Harald von Pein ${ }^{2}$,

Clemens Sommer ${ }^{2}$, Dorothea Haas ${ }^{3}$,

Konstanze Hörtnagel ${ }^{4}$, Saskia Biskup ${ }^{4}$

\section{Institute}

1 DKD Helios Klinik Wiesbaden

2 Institut für Neuropathologie, Universitätsmedizin Mainz

3 Zentrum Kinder- und Jugendmedizin, Dietmar-Hopp Stoffwechselzentrum, Universitätsklinikum Heidelberg,

4 CeGaT GmbH und Praxis für Humangenetik Tübingen

Korrespondenzadresse

Dr. med. Bertold Schrank

Fachbereich Neurologie

DKD Helios Klinik Wiesbaden

Aukammallee 33

65191 Wiesbaden

Bertold.Schrank@helios-kliniken.de

Bibliografie

DOI https://doi.org/10.1055/s-0043-107738 Klin Neurophysiol 2017; 96: 1-5

(c) Georg Thieme Verlag KG Stuttgart · New York ISSN 1434-0275 